Research Article

\title{
A Note on the Difference of Powers and Falling Powers
}

\author{
Taoufik Sabar (D) \\ Department of Mathematics and Computer Science, Faculty of Sciences Ben M'Sik, Hassan II University of Casablanca, \\ Casablanca, Morocco \\ Correspondence should be addressed to Taoufik Sabar; sabarsaw@gmail.com
}

Received 21 January 2021; Revised 4 March 2021; Accepted 19 March 2021; Published 5 April 2021

Academic Editor: Raúl E. Curto

Copyright ( 2021 Taoufik Sabar. This is an open access article distributed under the Creative Commons Attribution License, which permits unrestricted use, distribution, and reproduction in any medium, provided the original work is properly cited.

\begin{abstract}
Combinatorial sums and binomial identities have appeared in many branches of mathematics, physics, and engineering. They can be established by many techniques, from generating functions to special series. Here, using a simple mathematical induction principle, we obtain a new combinatorial sum that involves ordinary powers, falling powers, and binomial coefficient at once. This way, and without the use of any complicated analytic technique, we obtain a result that already exists and a generalization of an identity derived from Sterling numbers of the second kind. Our formula is new, genuine, and several identities can be derived from it. The findings of this study can help for better understanding of the relation between ordinary and falling powers, which both play a very important role in discrete mathematics.
\end{abstract}

\section{Introduction}

Identities involving binomial coefficients and combinatorial summations have several uses in mathematical physics and engineering and have appeared in several mathematical branches, such as combinatorics, probability theory, number theory, and graph theory. These identities can be deduced by many techniques, switching the order of summations for double sums and mathematical induction, forcing telescoping, and sometimes, it is possible to interpret an expression as counting some quantity or computing the probability of some events. But, there are also some more complicated analytic methods such as generating functions, inverse relations, integral representations, and special series like the gamma function or the beta functions $(c f)[1-3]$.

In this paper, we will put to use a new technique to obtain a new elegant identity involving ordinary powers, falling powers, and binomial coefficients. We use our main identity to convert from the difference of ordinary powers and falling powers to binomial coefficient. Then, we obtain some other sums as a consequence of our main identity, and one of them is a result that was already proven in [4].
Our work differs from previous studies on combinatorial sums because it uses a new and simple mathematical induction principle rather than the ordinary complex methods mentioned above.

First, we introduce our mathematical induction principle as a lemma, and then we put it to use to prove our main theorem. Afterwards, we prove our second main result from which, a formula that already exists in the literature can be induced.

\section{Main Results}

First, we introduce the following particular mathematical induction principle.

Lemma 1. Let $n \geq 1$, and consider $n$ assertions $A(1), \ldots, A(n)$. Suppose

(i) $A(n)$ is true.

(ii) $A(p) \Rightarrow A(p-1)$, for all $2 \leq p \leq n$.

Then, $A(p)$ is true for all $1 \leq p \leq n$.

Let $(n)_{p}$ denote the $p$ term falling factorial of $n$, $n(n-1), \ldots,(n-p+1)$. Now, we state our main result. 
Theorem 1. Let $n \geq 1$. Then

$$
\begin{aligned}
n^{p}-(n)_{p} & =\sum_{k=0}^{p-1}(-1)^{p-1-k} k^{p}\left(\begin{array}{l}
n \\
k
\end{array}\right)\left(\begin{array}{c}
n-k-1 \\
n-p
\end{array}\right) \\
& =(n)_{p}\left(1+\sum_{k=0}^{p-1}(-1)^{p-1-k} \frac{k^{p}}{k !(p-1-k) !(n-k)}\right),
\end{aligned}
$$

for all $p=1, \ldots, n$.

Proof. For $p=n$, let $S(n, p)$ denote Stirling numbers of the second kind. Since $S(p, p)=1$, we get

$$
\begin{aligned}
p ! & =\sum_{k=0}^{p}(-1)^{p-k} k^{p}\left(\begin{array}{l}
p \\
k
\end{array}\right) \\
& =p !\left(\frac{p^{p}}{p !}+\sum_{k=0}^{p-1}(-1)^{p-k} \frac{k^{p}}{k !(p-k-1) !(p-k)}\right),
\end{aligned}
$$

which implies

$$
\frac{p^{p}}{p !}=1+\sum_{k=0}^{p-1}(-1)^{p-1-k} \frac{k^{p}}{k !(p-k-1) !(p-k)} .
$$

Now suppose

$$
\frac{n^{p}}{(n)_{p}}=\left(1+\sum_{k=0}^{p-1}(-1)^{p-1-k} \frac{k^{p}}{k !(p-1-k) !(n-k)}\right) .
$$

We have

$$
\begin{aligned}
& \frac{n^{p}}{(n)_{p}}-1-\frac{n}{n-p+1} \sum_{k=0}^{p-2}(-1)^{p-2-k} \frac{k^{p-1}}{k !(p-2-k) !(n-k)} \\
& \quad=\sum_{k=0}^{p-1}(-1)^{p-1-k} \frac{k^{p}}{k !(p-1-k) !(n-k)}-\frac{n}{n-p+1} \sum_{k=0}^{p-2}(-1)^{p-2-k} \frac{k^{p-1}}{k !(p-2-k) !(n-k)} \\
& =\frac{1}{n-p+1}\left(\frac{(p-1)^{p}}{(p-1) !}+\sum_{k=0}^{p-2} \frac{(-1)^{p-1-k} k^{p}(n-p+1)+(-1)^{p-2-k} k^{p-1} n(p-1-k)}{k !(p-1-k) !(n-k)}\right) \\
& =\frac{1}{n-p+1}\left(\frac{(p-1)^{p}}{(p-1) !}+\sum_{k=0}^{p-2} \frac{(-1)^{p-1-k} k^{p-1}(k n-k p+k+n p-n-n k)}{k !(p-1-k) !(n-k)}\right) \\
& =\frac{1}{n-p+1}\left(\frac{(p-1)^{p}}{(p-1) !}+\sum_{k=0}^{p-2} \frac{(-1)^{p-1-k} k^{p-1}(p-1)}{k !(p-1-k) !}\right) \\
& =\frac{p-1}{n-p+1}=\frac{n-1}{n-p+1}-1 . \\
& \left.=\frac{p-1}{n-p+1} \frac{(-1)^{p-1-k}}{k !(p-1-k) !}\right) \\
& k=0
\end{aligned}
$$

where we used the fact that

$$
\sum_{k=0}^{p-1} \frac{(-1)^{p-1-k} k^{p-1}}{k !(p-1-k) !}=1
$$


Therefore,

$$
\begin{aligned}
& \frac{n^{p}}{(n)_{p}}-\frac{n}{n-p+1} \sum_{k=0}^{p-2}(-1)^{p-2-k} \frac{k^{p-1}}{k !(p-2-k) !(n-k)} \\
& \quad=\frac{n}{n-p+1},
\end{aligned}
$$

which implies

$$
\begin{aligned}
\left(1+\sum_{k=0}^{p-2}(-1)^{p-2-k} \frac{k^{p-1}}{k !(p-2-k) !(n-k)}\right) & =\frac{n-p+1}{n} \frac{n^{p}}{(n)_{p}} \\
& =\frac{n^{p-1}}{(n)_{p-1}}
\end{aligned}
$$

The next lemma is not hard to see, but necessary in the proof of our next result.

Lemma 2. Let $n \geq 1$,

$$
\sum_{k=0}^{n}(-1)^{n-k} \frac{k^{p}}{k !(n-k) !}=0
$$

for all $p=0, \ldots, n-1$.

Proof. For all $n \geq 1$, we have

$$
\begin{aligned}
0 & =\sum_{k=0}^{n}(-1)^{n-k} \frac{k^{n}}{k !(n-k) !}-\sum_{k=0}^{n-1}(-1)^{n-1-k} \frac{k^{n-1}}{k !(n-1-k) !} \\
& =\frac{n^{n}}{n !}+\sum_{k=0}^{n-1}(-1)^{n-k} \frac{k^{n}+(n-k) k^{n-1}}{k !(n-k) !} \\
& =n \sum_{k=0}^{n}(-1)^{n-k} \frac{k^{n-1}}{k !(n-k) !}
\end{aligned}
$$

Similarly,

$$
\sum_{k=0}^{n-1}(-1)^{n-1-k} \frac{k^{n-2}}{k !(n-1-k) !}=0 .
$$

Then,

$$
\begin{aligned}
0 & =\sum_{k=0}^{n}(-1)^{n-k} \frac{k^{n-1}}{k !(n-k) !}-\sum_{k=0}^{n-1}(-1)^{n-1-k} \frac{k^{n-2}}{k !(n-1-k) !} \\
& =n \sum_{k=0}^{n}(-1)^{n-k} \frac{k^{n-2}}{k !(n-k) !} .
\end{aligned}
$$

With the assumption that $0^{\circ}=1$ of course, it suffices to repeat the same process $n$ times.

Theorem 2. Let $n \geq 1$ and $p=0, \ldots, n-1$. Then

$$
\frac{n^{p}}{(n)_{p+q}}=\sum_{k=1}^{p+q-1}(-1)^{p+q-1-k} \frac{k^{p}}{k !(p+q-1-k) !(n-k)},
$$

for all $q=1, \ldots, n-p$

Proof. For $q=n-p$, we get

$$
\frac{n^{p}}{(n)_{n}}=\sum_{k=0}^{n-1}(-1)^{n-1-k} \frac{k^{p}}{k !(n-1-k) !(n-k)},
$$

as an immediate consequence of the previous lemma. Now, suppose

$$
\frac{n^{p}}{(n)_{p+q}}=\sum_{k=0}^{p+q-1}(-1)^{p+q-1-k} \frac{k^{p}}{k !(p+q-1-k) !(n-k)} .
$$

We get,

$$
\begin{aligned}
& \frac{n^{p}}{(n)_{p+q-1}}-\sum_{k=0}^{p+q-2}(-1)^{p+q-2-k} \frac{k^{p}}{k !(p+q-2-k) !(n-k)} \\
& =(n-p-q+1) \frac{n^{p}}{(n)_{p+q}}-\sum_{k=0}^{p+q-2}(-1)^{p+q-2-k} \frac{k^{p}}{k !(p+q-2-k) !(n-k)} \\
& =(n-p-q+1) \sum_{k=0}^{p+q-1}(-1)^{p+q-1-k} \frac{k^{p}}{k !(p+q-1-k) !(n-k)}-\sum_{k=0}^{p+q-2}(-1)^{p+q-2-k} \overline{k !(p+q-2-k) !(n-k)} \\
& =\frac{(p+q-1)^{p}}{(p+q-1) !}+\sum_{k=0}^{p+q-2} \frac{(-1)^{p+q-1-k}\left(k^{p}[(n-p-q+1)+(p+q-1-k)]\right)}{k !(p+q-1-k) !(n-k)} \\
& =\sum_{k=0}^{p+q-1}(-1)^{p+q-1-k} \frac{k^{p}}{k !(p+q-1-k) !}=0 .
\end{aligned}
$$


As a direct consequence of the previous theorem, we get the following identity.

Corollary 1 (see Theorem 5.1 of [4]). If we take $p=0$ in the last theorem, we get

$$
\frac{1}{(n)_{q}}=\sum_{k=0}^{q-1}(-1)^{q-1-k} \frac{1}{k !(q-1-k) !(n-k)},
$$

which means

$$
\frac{1}{n\left(\begin{array}{c}
n-1 \\
q-1
\end{array}\right)}=\sum_{k=0}^{q-1}(-1)^{q-1-k} \frac{\left(\begin{array}{c}
q-1 \\
k
\end{array}\right)}{(n-k)}
$$

\section{Data Availability}

No data were used to support this study.

\section{Conflicts of Interest}

The authors declare that they have no conflicts of interest.

\section{References}

[1] H. W. Gould, Combinatorial Identities, Morgantown Printing and Binding Co., Morgantown, WV, USA, 1972.

[2] T. Mansour, "Combinatorial identities and inverse binomial coefficients," Advances in Applied Mathematics, vol. 28, no. 2, pp. 196-202, 2002.

[3] Y. Simsek, "Combinatorial sums and binomial identities associated with the beta-type polynomials," Hacettepe Journal of Mathematics and Statistics, vol. 47, no. 5, pp. 1144-1155, 2018.

[4] Y. Simsek, "Beta-type polynomials and their generating functions," Applied Mathematics and Computation, vol. 254, pp. 172-182, 2015. 